\title{
A Simple Homemade Electrospinning for Nanoscale Fibres Production
}

\author{
Ngurah Ayu Ketut Umiati ${ }^{1,}$, V.G.S. Kadarisman ${ }^{1}$, Agus Subagio $^{1}$, Kunto Wandono ${ }^{2}$ \\ ${ }^{1}$ Physics Department, Faculty of Sciences and Mathematics, Diponegoro University, Semarang - Indonesia \\ ${ }^{2}$ Hakaku, Commanditaire Vennootschap, Yogyakarta, Indonesia
}

\begin{abstract}
A high technology often is required by electrospinning device to produce a nanoscale fibre. This requirement results in difficulties to get the device simple design and operate with reasonable price. This paper presents the work to implement design of the electrospinning system. This electrospinning system consists of a main construction body, an electrical power supply to provide a voltage source direct current form, a syringe pump and a flat collector. The main body are made of acrylic with knockdown construction since acrylic is an easy material to shape. The power supply has a simple main single switch with high ferite transformer to produce high dc voltage through a diode - capacitor filtering. A used medical syringe has been modified as an injection pump to spray the materials into fibre form. A simple flat metal has been reconstructed to be electrode - ground collector to receive fibre product. The test was carried out using polyaniline materials. The general parameters in the production process are resolution of the spraying rate $\mu \ell /$ minute and the power supply provide electricity in kiloVolt. The result of fibre production observed by using SEM shows that the electrospinning device successfully produces fibres on a nanometer scale.
\end{abstract}

Keywords: Electrospinning, Nanofibre, Synthesize

\section{Introduction}

Electrospinning is a spinning technique using an electrostatic force approach to produce fibre from a polymer solution or fused. The fibres produced from this method have a diameter range from nano to micrometers and a wider surface area than conventional spinning methods[1]. This method is in great demand because it can control production, fibre structure, porosity, orientation and dimensions of the fibre produced [2].

The electrospinning method is very effective for producing nanoscale fibres using polymer solutions as bait [3][4]. However, this process is strongly influenced by various factors including the distance of the capillary pipe (spinneret) to the collector plate made of metal, the needle diameter on the spinneret used, the viscosity of the solution, and the supply of high voltage used [5]. Those influence factors have made the design of electrospinning must be carried out by using a high technology manufacturing process.

This electrospinning method has also been used to make polyaniline nanofibre. Shahi et.al (2011) add poly (vinyl alcohol) (PVA) to polyaniline obtained from oxidative chemical polymerization to produce polyaniline nanofibre using the electrospinning method [6]. The addition of biosensor amperometric on nanofibre polyaniline was also carried out using the electrospinning method. The previous polyaniline nanofibre was also obtained from the electrospinning method [7]. The use of the electrospinning method to obtain nanofibre polyaniline continues to grow, including for humidity sensor applications [8], tissue culture of human adipose-derived stem cells (hASCs) [9] and sensors [10].

The main part of the electrospinning device consists of a high-voltage direct current (DC) source, a spinneret tube, a small diameter needle, and a metal collector plate [4, 11]. Positive electrodes are connected to the tip of the needle and negative electrodes are connected to the fibre collector or vice versa. The positive end of the voltage source is connected to the tip of the tip so that the solution will be positively charged. The negative end (ground) of the voltage source is connected to the collector's part, as a place to capture the formed fibre.

In this work, the design of electrospinning equipment for fabricating nanofibre material was carried out. Electrospinning equipment made with a syringe pump design can be filled by more than one syringe. The type of collector used is a collector stand. The development of an electrospinning system is carried out using raw materials and local components on the market. The material used for testing is polyaniline (PANI) with a mixture of polyvinyl alcohol (PVA). Characterization of the nanofibre morphological structure was carried out using Scanning Electron Microscopy (SEM). Electrospinning that has been made is expected to produce nanofibre material that can be applied in various fields

* Corresponding author: ngurahayuketutumiati@gmail.com 


\section{Method}

Stages of research include the preparation stage, the design and manufacture stages, and the testing phase.

\section{a. Preparation stage}

Preparation stage in the form of preparation of materials and equipment needed. Some of the main ingredients used are high voltage power supply, syringe pump covering permanent magnet DC motor, motor controller, mechanic part, and cashing, collector part including permanent magnet DC motor, motor controller, cashing and wiring, casing part made from acrylic material, and test material covering polyaniline, PVA and distilled water

b. Design and manufacture stages

The electrospinning device contains a spinneret made from a type of hypodermic syringe needle connected to an electric source, syringe pumps, and grounded collector. Figure 1 below shows the electronic circuit of electrospinning device.

\section{c. Testing phase}

There are three steps in the fabrication of nanofibre polymers using the electrospinning method. Those steps are (i) converting doped polymers to dedoped polymers, (ii) making electrospinning feed solutions and (iii) electrospinning processes. The polymer material used is PANI which is doped with $1 \mathrm{M} \mathrm{HCl}$ and PVA. Scanning Electro Microscopy (SEM) was used to observe the fibre product.

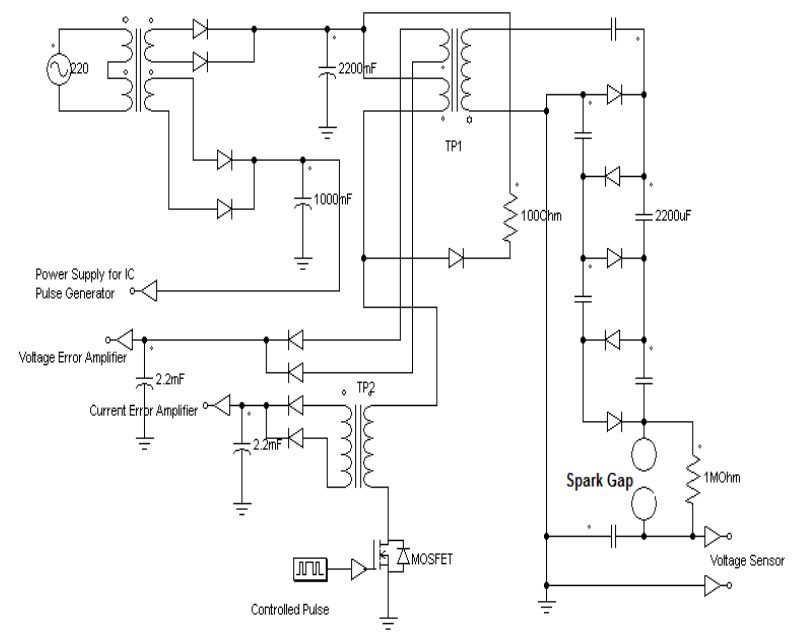

Fig. 1. High Voltage electronic circuit for electrospinning device.

\section{Results and Discussion}

In this chapter, the implementation of design scheme in Figure 1 is provided. The production of polyaniline nanofibre is presented as the output of electrospinning.

\subsection{Implementation of Design}

The design and manufacture of electrospinning machines have been successfully made. This device consists of an automatic injection pump and an electrical power source that is applied to a sample of polymer solutions to make small diameter fibres. The power supply took voltage from the grid in 220 Volt and converted the DC output voltage in the ranges of $0-30 \mathrm{kV}$. Figure 2 shows the power supply for electrospinning device.

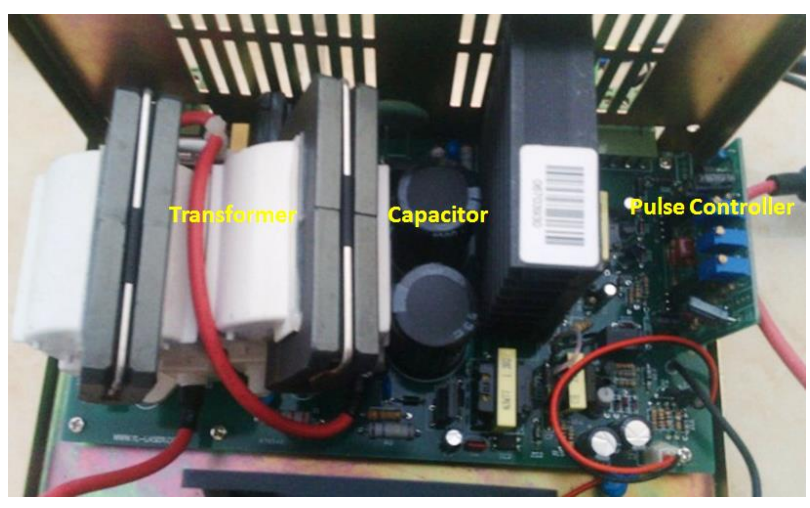

Fig. 2. Electric power supply electrospinning machine.

An injection pump made from medical syringe is as a solution sprayer injection pump with a controlled flow rate. Syringe pump flow rates can be adjusted in the range of $100 \mathrm{~L} /$ minute to $600 \mathrm{~L} /$ minute. The standard volume syringe is $10 \mathrm{~mL}$ with an installed capacity of about 3 syringes as it is shown in Figure 3.

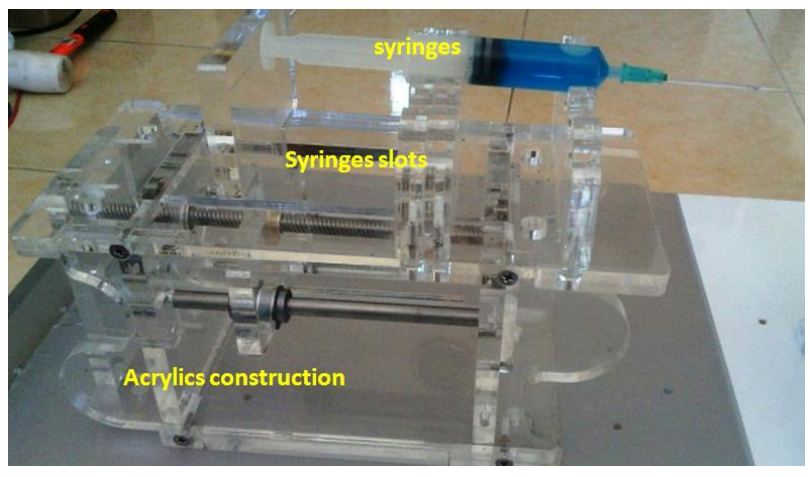

Fig. 3. Syringe electrospinning pump.

Collector was made in the form of flat metal material with a distance setting of $3-20 \mathrm{~cm}$. Figure 4 shows the shape of the collector.

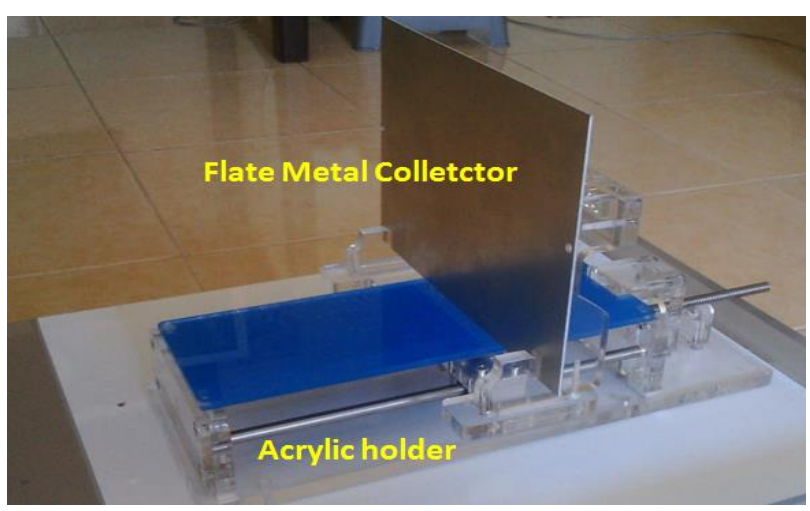

Fig. 4. Electrospinning device collector. 


\subsection{Fibre Material as A sample of Nanofibre Product}

During initial preparation for production test, a Polyvinyl Alcohol (PVA) was mixed with aquadestilata $\left(\mathrm{H}_{2} \mathrm{O}\right)$ with a ratio of 1:10 (wt \%). Preparations of the prepared ingredients were inserted into the syringe for electrospinning. The spraying process was executed at a speed of $6 \mu \ell /$ minute and the positive high voltage pole was connected to the needle syringe. The electrostatic field of the collector connected to the negative pole sprayed the solution until the product becomes a smallsized fibre. Figure 5 shows the results of a macro PVA fibre photo using an SEM.

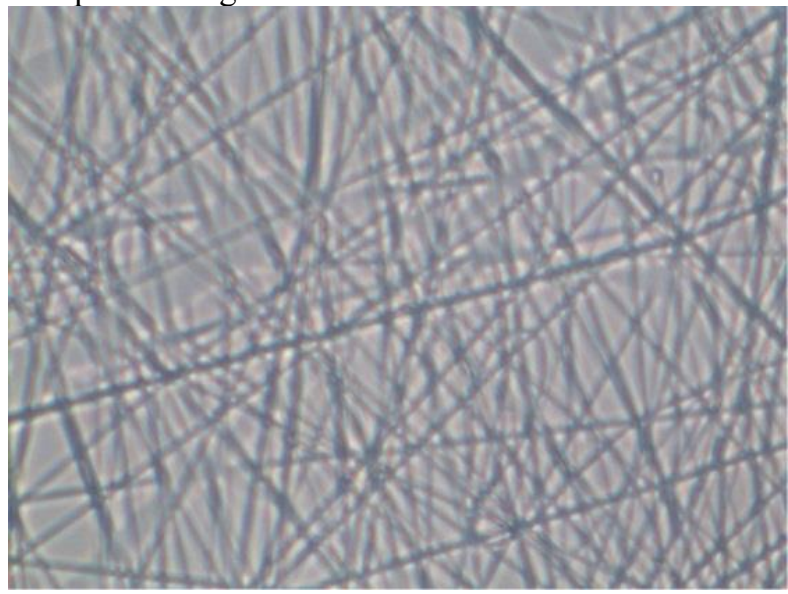

Fig. 5. Photograph of PVA electrospinning 1:10 (wt\%)..

In the next fibre material test, polyaniline is used. Polyaniline solution is made by mixing polyaniline into DMSO solvent. To facilitate the solubility of polyaniline nanofibre in DMSO solvents, the influence of $\mathrm{HCl}$ must be removed. The effect of $\mathrm{HCl}$ was removed by converting polyaniline from doped polyaniline to undoped by washing using $\mathrm{NH}_{3}$ solution [11]. In this process, the $\mathrm{NH}_{3}$ molar used is proportional to the molar dopant $\mathrm{HCl}$ used. After obtaining undoped polyaniline, a $5 \%$ wt polyaniline solution was made with DMSO solvent. The feed solution was a PVA solution with a concentration of $10 \%$ wt. In the next process, a $5 \%$ polyaniline-DMSO solution is mixed with $10 \%$ PVA. The electrospinning process was carried out by using constant injection voltage at $15 \mathrm{kV}$ and a syringe needle of $0.5 \mathrm{~mm}$ for 30 minutes. The distance between the tip of the needle and the collector is $11 \mathrm{~cm}$ and the length of the needle is $1 \mathrm{~cm}$.

Figure 6 below shows the results of electrospun polyaniline nanofibre observed by SEM.

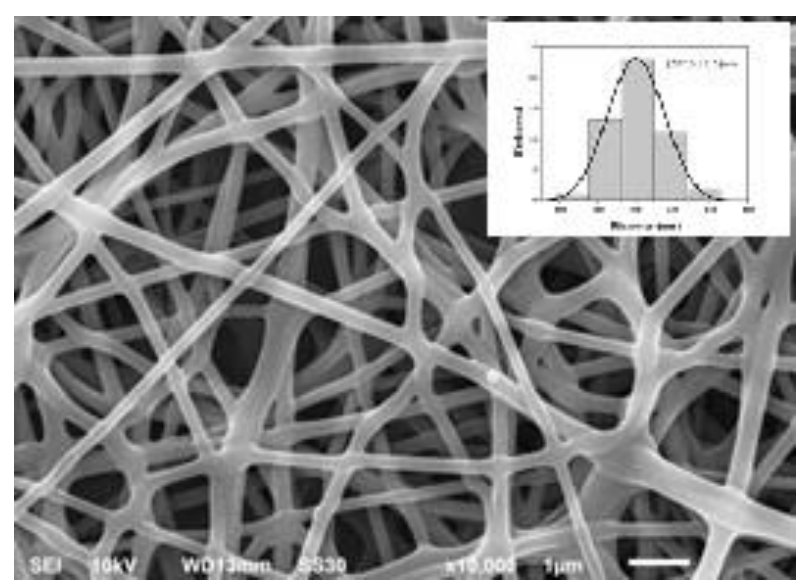

Fig. 6. Results of SEM electrospun polyaniline with a dopant concentration of $\mathrm{HCl} 1 \mathrm{M}$.

From the results above it can be seen that the general parameters needed for the manufacture of electrospinning machines are resolution of the spraying rate of the solution in $\mu \ell /$ minute and the amount of high voltage power source in kiloVolt $(\mathrm{kV})$. The homemade electrospinning has successfully provided the rate of spraying the solution in $\mu \ell /$ minute and the injected voltage in order of kilo Volt to produce fibre morphology in nanometer diameter

\section{Conclusion}

The electrospinning system has been implemented with the main body in the form of an acrylic chamber, an electrical power supply to generate a constant dc high voltage dc, an adjustable syringe pump, and flat collector. Electrospinning tests have been successfully carried out by using polymer material. The main parameter such as the spraying rate of the solution in $\mu \ell$ / minute and constant injected voltage in kiloVolt for producing nanofibre has been provided by the proposed electrospinning device. Observation using SEM shows that the fibre produced by electrospinning device in this work was in a nanometer scale

Our thanks go to DIPA PNBP 2018 budget year with an agreement contract Number: 1756 I /UN7.5.8/PG/2018 which has funded this research.

\section{References}

1. N. Bhardwaj, S.C. Kundu, Electrospinning: A fascinating fibre fabrication technique, Biotechnol. Adv. 28 (2010)

2. A. Baji, Y.W. Mai, S.C. Wong, M. Abtahi, P. Chen, Electrospinning of polymer nanofibres: Effects on oriented morphology, structures and tensile properties, Compos. Sci. Technol. 70: 703-718 (2010)

3. R. Sood, S. Cavaliere, D.J. Jones, J. Rozière, Electrospun nanofibre composite polymer electrolyte fuel cell and electrolysis membranes, Nano Energy. 26: 729-745 (2016) 
4. M. Alazab, G.R. Mitchell, F.J. Davis, S.D. Mohan, Sustainable Electrospinning of Nanoscale Fibres, Procedia Manuf. 12: 66-78 (2017)

5. R.B. Kaner, Synthesis and applications of conducting polymer nanofibres, MRS Bull. 41: 785790 (2016)

6. M. Shahi, A. Moghimi, B. Naderizadeh, B. Maddah, Electrospun PVA-PANI and PVA-PANIAgNO3composite nanofibres, Sci. Iran. 18: 13271331 (2011)

7. Y.J. Shin, J. Kameoka, Amperometric cholesterol biosensor using layer-by-layer adsorption technique onto electrospun polyaniline nanofibres, J. Ind. Eng. Chem. 18: 193-197 (2012)

8. Q. Lin, Y. Li, M. Yang, Polyaniline nanofibre humidity sensor prepared by electrospinning, Sensors Actuators, B Chem. 161 (2012).

9. N. Abdul Rahman, V. Feisst, M.E. Dickinson, J. Malmström, P.R. Dunbar, J. Travas-Sejdic, Functional polyaniline nanofibre mats for human adipose-derived stem cell proliferation and adhesion, Mater. Chem. Phys. 138: 333-341 (2013)

10. Y.A. Ismail, J.G. Martínez, T.F. Otero, Fibroin/Polyaniline microfibrous mat. Preparation and electrochemical characterization as reactive sensor, Electrochim. Acta. 123: 501-510 (2014)

11. P. Wen, M.H. Zong, R.J. Linhardt, K. Feng, H. Wu, Electrospinning: A novel nano-encapsulation approach for bioactive compounds, Trends Food Sci. Technol. 70: 56-68 (2017) 